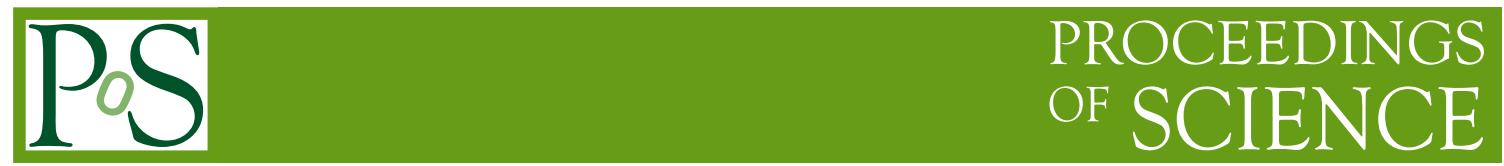

\title{
Exclusive production of Higgs boson, b-antib and gluonic jets
}

Antoni SZCZUREK*

Rzeszow University, Poland

Institute of Nuclear Physics, Poland

E-mail: antoni.szczurek@ifj.edu.pl

We discuss exclusive central production of Higgs boson, quark-antiquark and digluon dijets. Several differential distributions are shown and disussed. Irreducible leading-order $b \bar{b}$ background to Higgs production is calculated in several kinematical variables. The signal-to-background ratio is shown and several improvements are suggested by imposing cuts on $b(\bar{b})$ transverse momenta and rapidities.

The 2011 Europhysics Conference on High Energy Physics - HEP2011

July 21-27, 2011

Grenoble, Rhône-Alpes, France

${ }^{*}$ Speaker. 


\section{Introduction}

Khoze, Martin and Ryskin developed a QCD approach for exclusive production of Higgs boson [1]. The approach can be easily generalized to other exclusive processes. Recently we have applied this approach to Standard Model Higgs boson, quark-antiquark and digluon exclusive production $[2,3,4,5]$.

Since the cross section for exclusive Higgs boson production is rather small, only $b \bar{b}$ final state can be used to identify Higgs boson. This means that a $b \bar{b}$ continuum background is of crucial importance. We discuss this irreducible background here.

In our calculations we include exact matrix elements and do full four-body calculations for all considered processes. The kinematically complete calculations allow to include any cut on kinematical variables which is very usefull in order to find the Higgs boson signal.

\section{Formalism}

Let us concentrate on the simplest case of the production of $q \bar{q}$ pair in the color singlet state. Color octet state would demand an emission of an extra gluon which considerably complicates the calculations. We do not consider the $q \bar{q} g$ contribution as it is higher order compared to the one considered here.

We write the amplitude of the exclusive diffractive $q \bar{q}$ pair production $p p \rightarrow p(q \bar{q}) p$ in the color singlet state as

$$
\mathscr{M}_{\lambda_{q} \lambda_{\bar{q}}}^{p p \rightarrow p p q \bar{q}}\left(p_{1}^{\prime}, p_{2}^{\prime}, k_{1}, k_{2}\right)=s \cdot \pi^{2} \frac{1}{2} \frac{\delta_{c_{1} c_{2}}}{N_{c}^{2}-1} \mathfrak{J} \int d^{2} q_{0, t} V_{\lambda_{q} \lambda_{\bar{q}}}^{c_{1} c_{2}}\left(q_{1}, q_{2}, k_{1}, k_{2}\right) \frac{f_{g, 1}^{\mathrm{off}}\left(x_{1}, x_{1}^{\prime}, q_{0, t}^{2}, q_{1, t}^{2}, t_{1}\right) f_{g, 2}^{\mathrm{off}}\left(x_{2}, x_{2}^{\prime}, q_{0, t}^{2}, q_{2, t}^{2}, t_{2}\right)}{q_{0, t}^{2} q_{1, t}^{2} q_{2, t}^{2}} .
$$

where $\lambda_{q}, \lambda_{\bar{q}}$ are helicities of heavy $q$ and $\bar{q}$, respectively. Above $f_{1}^{\text {off }}$ and $f_{2}^{\text {off }}$ are the off-diagonal unintegrated gluon distributions in nucleon 1 and 2 , respectively. The longitudinal momentum fractions of active gluons are calculated based on kinematical variables of outgoing quark and antiquark. The bare amplitude above is subjected to absorption corrections. The absorption corrections are taken here in a simple multiplicative form.

The color singlet $q \bar{q}$ pair production amplitude can be written as [4]

$$
V_{\lambda_{q} \lambda_{\bar{q}}}^{c_{1} c_{2}}\left(q_{1}, q_{2}, k_{1}, k_{2}\right) \equiv n_{\mu}^{+} n_{v}^{-} V_{\lambda_{q} \lambda_{\bar{q}}}^{c_{1} c_{2}, \mu v}\left(q_{1}, q_{2}, k_{1}, k_{2}\right)
$$

The tensorial part of the amplitude reads:

$$
V_{\lambda_{q} \lambda_{\bar{q}}}^{\mu v}\left(q_{1}, q_{2}, k_{1}, k_{2}\right)=g_{s}^{2} \bar{u}_{\lambda_{q}}\left(k_{1}\right)\left(\gamma^{v} \frac{\hat{q}_{1}-\hat{k}_{1}-m}{\left(q_{1}-k_{1}\right)^{2}-m^{2}} \gamma^{\mu}-\gamma^{\mu} \frac{\hat{q}_{1}-\hat{k}_{2}+m}{\left(q_{1}-k_{2}\right)^{2}-m^{2}} \gamma^{v}\right) v_{\lambda_{\bar{q}}}\left(k_{2}\right)
$$

The coupling constants $g_{s}^{2} \rightarrow g_{s}\left(\mu_{r, 1}^{2}\right) g_{s}\left(\mu_{r, 2}^{2}\right)$. In the present calculation we take the renormalization scale to be $\mu_{r, 1}^{2}=\mu_{r, 2}^{2}=M_{q \bar{q}}^{2}$. The exact matrix element is calculated numerically. Analytical formulae are shown explicitly in [4].

The off-diagonal parton distributions $(\mathrm{i}=1,2)$ are calculated as

$$
f_{i}^{\mathrm{KMR}}\left(x_{i}, Q_{i, t}^{2}, \mu^{2}, t_{i}\right)=\left.R_{g} \frac{d\left[g\left(x_{i}, k_{t}^{2}\right) S_{1 / 2}\left(k_{t}^{2}, \mu^{2}\right)\right]}{d \log k_{t}^{2}}\right|_{k_{t}^{2}=Q_{i t}^{2}} F\left(t_{i}\right),
$$



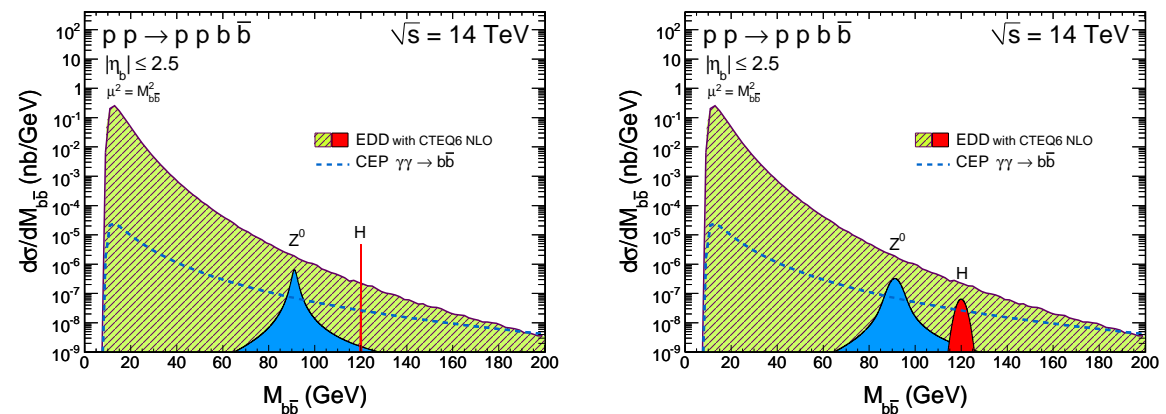

Figure 1: The $b \bar{b}$ invariant mass distribution for $\sqrt{s}=14 \mathrm{TeV}$ and for $b$ and $\bar{b}$ jets for $-2.5<\eta<2.5$ corresponding to the ATLAS detector. The left panel shows purely theoretical predictions, while the right panel includes experimental effects due to experimental uncertainty in missing mass measurement.

where $S_{1 / 2}\left(q_{t}^{2}, \mu^{2}\right)$ is a Sudakov-like form factor relevant for the case under consideration. It is reasonable to take a factorization scale as: $\mu_{1}^{2}=\mu_{2}^{2}=M_{q \bar{q}}^{2}$.

The factor $R_{g}$ here cannot be calculated from first principles but can be estimated in the case of off-diagonal collinear PDFs when $x^{\prime} \ll x$ and $x g=x^{-\lambda}(1-x)^{n}$. Typically $R_{g} \sim 1.3-1.4$ at the Tevatron energy. The off-diagonal form factors are parametrized here as $F(t)=\exp \left(B_{\text {off }} t\right)$. In practical calculations we take $B_{\text {off }}=2 \mathrm{GeV}^{-2}$. In evaluating $f_{1}$ and $f_{2}$ needed for calculating the amplitude (2.1) we use different collinear distributions.

\section{Results}

In our papers we have calculated differential cross sections not only for exclusive Higgs boson production but also for $b \bar{b}$ and $g g$. In all our calculations we take into account off-shellness of the gluons initiating a relevant hard subprocess. The details about the off-shell matrix element for Higgs boson production can be found in Ref. [6]. In contrast to the exclusive production of $\chi_{c}$ mesons, due to a large factorization scale $\sim M_{H}$, the off-shell effects for $g^{*} g^{*} \rightarrow H$ give only a few percent change compare to the calculation with on-shell matrix elements used in the literature. We use the same unintegrated gluon distributions for Higgs, continuum $b \bar{b}$ and digluon production.

The Higgs boson differential cross sections are calculated assuming a three-body process $p p \rightarrow p H p$. Assuming full coverage for outgoing protons we construct two-dimensional distributions $d \sigma / d y d^{2} p_{t}$ in Higgs rapidity and transverse momentum. The distribution is used then in a simple Monte Carlo code which includes the Higgs boson decay into the $b \bar{b}$ channel. It is checked subsequently whether $b$ and $\bar{b}$ enter into the central detector.

In the left panel of Fig.1 we show the double diffractive contribution for CTEQ6 [7] collinear gluon distribution and the contribution from the decay of the Higgs boson including natural decay width calculated as in Ref. [8], see the sharp peak at $M_{b \bar{b}}=120 \mathrm{GeV}$. The phase space integrated cross section for the Higgs production, including absorption effects with gap survival probability $S_{G}=0.03$ is less than $1 \mathrm{fb}$. The result shown in Fig. 1 includes branching fraction for $\mathrm{BR}(H \rightarrow$ $b \bar{b}) \approx 0.8$ and the rapidity restrictions. The much broader Breit-Wigner type peak to the left of the Higgs signal corresponds to the exclusive production of the $Z^{0}$ boson with the cross section 

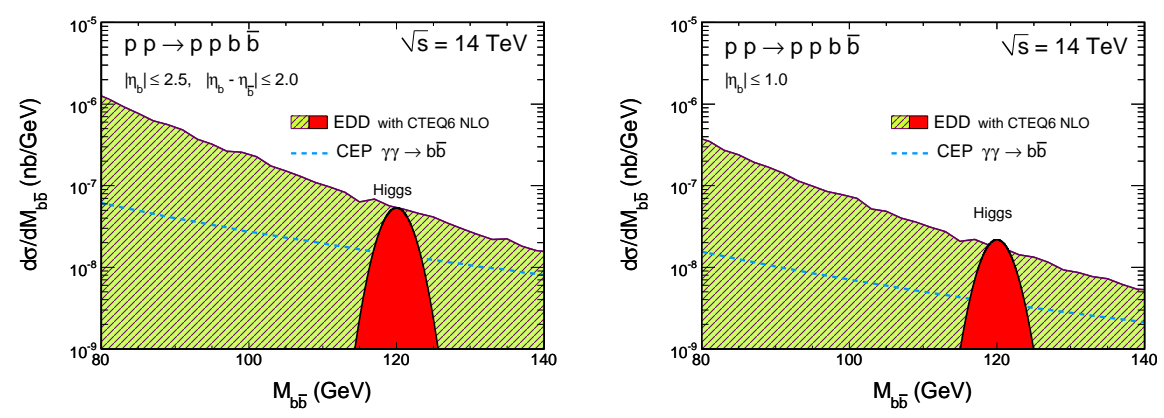

Figure 2: The $b \bar{b}$ invariant mass distribution for $\sqrt{s}=14 \mathrm{TeV}$. In the left panel the rapidity difference is limited to $(-1,1)$ and in the right panel both pseudorapidities are $-1<\eta<1$.

calculated as in Ref. [9]. The branching fraction $\mathrm{BR}\left(Z^{0} \rightarrow b \bar{b}\right) \approx 0.15$ has been included in addition. In contrast to the Higgs case the absorption effects for the $Z^{0}$ production are much smaller [9]. The sharp peak corresponding to the Higgs boson clearly sticks above the background.

In reality the situation is, however, much worse as both protons and in particular $b$ and $\bar{b}$ jets are measured with a certain precision which automatically leads to a smearing in $M_{b \bar{b}}$. Experimentally instead of $M_{b \bar{b}}$ one will measure rather two-proton missing mass $\left(M_{p p}\right)$. In our calculations the experimental effects are included in the simplest way by a convolution of the theoretical distributions with the Gaussian smearing function with $\sigma=2 \mathrm{GeV}$ which is determined mainly by the precision of measuring forward protons. In the right panel we show the two-proton missing mass distribution when the experimental smearing is included. Now the bump corresponding to the Higgs boson is below the $b \bar{b}$ background.

In Refs. [3, 4] we have discussed in great detail how to improve the difficult situation. Examples are shown in Fig.2. In the left panel we show the situation when a cut on pseudorapidiy difference is limited to the interval $(-1,1)$ and in the right panel when cuts on rapidity of $b$ and $\bar{b}$ are imposed. In both cases the situation seems much better. We have checked, however, that this is an optimal situation and further imporovement of the signal-to-background ratio is not possible.
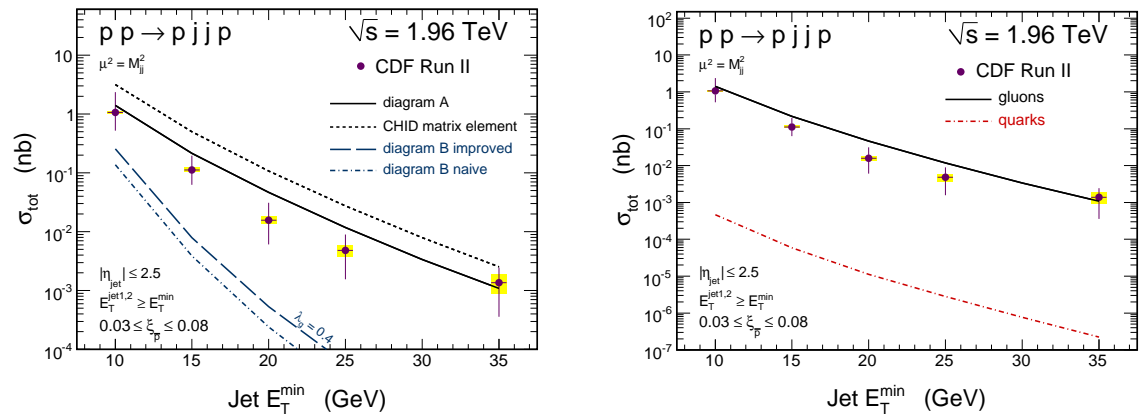

Figure 3: The total cross section as a function of $E_{t, \min }$. The experimental data points are taken from Ref. [12]. Left panel: standard digluon contribution for diagam A with our matrix element (solid line) and CHID matrix element (short-dashed line) and a new contribution (long-dashed line) not discussed here. Right panel: quark-antiquark (dash-dotted line) contribution. 
Now we come to the distributions of dijets. The details can be found in [5]. In Fig.3 we show the total cross section as a function of minimal $E_{T}$. Already the gluonic contribution (thick solid line) is slightly above the data. In the case of quark-antiquark dijets we present the contribution of $u \bar{u}, d \bar{d}, s \bar{s}, c \bar{c}$ and $b \bar{b}$. In the first three cases, we put the quark masses to zero, and in the last two cases we take explicit masses known from the phenomenology. The sum of all quark-antiquark contributions is shown in the right panel by the dash-dotted curve. We conclude that the quarkantiquark jet contribution is smaller by more than two orders of magnitude than the digluon one. The rough agreement with the experimental data gives confidence to the calculation of the exclusive Higgs boson production.

\section{Conclusions}

We have shown and discussed differential distributions for the continuum $b \bar{b}$ production. Inclusion of the experimental resolution is necessary when comparing the Higgs signal and the $b \bar{b}$ background. Our analysis shows that a special cuts can be useful to see a signal above the background.

Our analysis indicates that a real experiment can be rather difficult. The situation could be better for some scenarios beyond the Standard Model [10, 11].

I am indebted to Rafał Maciuła and Roman Pasechnik for collaboration on the issues presented here. I congratulate Johann Collot and collaborators for very good organization and friendly atmosphere during EPS2011.

\section{References}

[1] V. A. Khoze, A. D. Martin and M. G. Ryskin, Phys. Lett. B 401, 330 (1997);

A. B. Kaidalov, V. A. Khoze, A. D. Martin and M. G. Ryskin, Eur. Phys. J. C 33, 261 (2004).

[2] R. Maciuła, R. Pasechnik and A. Szczurek, Phys. Lett. B 685, 165 (2010).

[3] R. Maciuła, R. Pasechnik and A. Szczurek,arXiv:1006.3007 [hep-ph], Phys. Rev. D82 114011 (2010).

[4] R. Maciuła, R. Pasechnik and A. Szczurek,arXiv:1011.5842 [hep-ph]. Phys. Rev. D83 114034 (2011).

[5] R. Maciuła, R. Pasechnik and A. Szczurek, arXiv:1109.5517 [hep-ph].

[6] R. S. Pasechnik, O. V. Teryaev and A. Szczurek, Eur. Phys. J. C 47, 429 (2006).

[7] J. Pumplin et al., JHEP 0207, 012 (2002).

[8] G. Passarino, Nucl. Phys. B 488, 3 (1997).

[9] A. Cisek, W. Schäfer and A. Szczurek, Phys. Rev. D80 074013 (2009).

[10] B. E. Cox, F. K. Loebinger, A. D. Pilkington, JHEP 0710, 090 (2007).

[11] S. Heinemeyer, V.A. Khoze, M.G. Ryskin, W.J. Stirling, M. Tasevsky, and G. Weiglein, Eur. Phys. J. C53 231 (2008).

[12] T. Aaltonen et al. [CDF Collaboration], Phys. Rev. D 77, 052004 (2008);

A. A. Affolder et al. [CDF Collaboration], Phys. Rev. Lett. 88, 151802 (2002);

A. A. Affolder et al. [CDF Collaboration], Phys. Rev. Lett. 85, 4215 (2000). 